\section{CLINICAL REPORT}

\begin{abstract}
Q. Chen
X.-Z. Chen

J.-M. Wang

S.-W. Li

T. Jiang

J.-P. Dai

SUMMARY: Intracranial meningeal hemangiopericytomas in children and adolescents are prone to bleeding during surgery. CT and MR imaging may serve a role in preoperative diagnosis. The purpose of this report was to describe the radiologic findings in 9 pathologically proved cases of intracranial meningeal hemangiopericytomas in children and adolescents. The average duration of symptoms was short (mean, 4.0 months; median, 1.5 months). The intraoperative blood loss was large (mean, 3561 $\mathrm{mL}$; median, $1000 \mathrm{~mL}$ ). Tumors were extra-axial, irregularly multilobular, and hypervascular. Radiologically, they showed mixed attenuation on precontrast CT images, heterogeneous signal intensity on precontrast MR imaging, and marked and heterogeneous enhancement on postcontrast MR imaging. Bone erosion was sometimes present, but hyperostosis was not present. On MR imaging, multiple signal intensity voids of vessels were observed in $100 \%$ (8/8) of tumors with AVM-like signal intensity flow voids in $25 \%(2 / 8)$ of tumors. These results suggest that CT and MR imaging findings may be helpful for preoperative diagnosis.
\end{abstract}

\section{Intracranial Meningeal Hemangiopericytomas in Children and Adolescents: CT and MR Imaging Findings}

ABBREVIATIONS: AT/RT = atypical teratoid/rhabdoid tumor; Gd-DTPA = gadolinium-diethylenetriamine pentaacetic acid; PNET = primitive neuroectodermal tumor; $\mathrm{WHO}=$ World Health Organization
$\mathbf{H}^{\mathrm{e}}$ emangiopericytoma was first described by Stout and Murray $^{1}$ in 1942. Hemangiopericytoma is a rare tumor with an incidence of less than $1 \%$ of all CNS tumors, ${ }^{2}$ with only $10 \%$ of cases occurring in children. ${ }^{3}$ The average age at first presentation ranges from 35 to 44 years, ${ }^{2,4}$ with patient ages from 2 days $^{5}$ to 86 years. ${ }^{6}$ Hemangiopericytomas occurring in infants have a better prognosis than those in adults. ${ }^{3}$ It has been recommended that treatment consist of aggressive surgical resection followed by high-dose (50 Gy) external radiation therapy and long-term follow-up, because hemangiopericytoma is an aggressive tumor with a malignant potential and tends to recur and occasionally metastasizes, mainly to bone, lung, and liver. ${ }^{7}$ It is notable that the average blood loss among patients with preoperative embolization is much less than among those not receiving any embolization. ${ }^{7}$ Intracranial hemangiopericytomas are multilobulated extra-axial hypervascular tumors and are sometimes associated with narrow-based dural attachment and bone erosion, though hyperostosis is not present. Unlike with meningiomas, hemangiopericytomas show heterogeneous hyperattenuation on unenhanced CT scans, heterogeneous isointensity on

\section{Received March 7, 2011; accepted after revision May 2.}

From the Departments of Radiology (Q.C., X.-Z.C., S.-W.L., J.-P.D.), Neuropathology (J-M.W.), and Neurosurgery (T.J.), Beijing Tiantan Hospital, Capital Medical University, Beijing, China; and Neuroimaging Center (O.C., S.-W.L., J.-P.D.), Beijing Neurosurgical Institute, Beijing, China.

This study was sponsored by National Basic Research Program of China (973 Program, project 2011CB707804).

Please address correspondence to Jianping Dai, MD, Department of Radiology, Beijing Tiantan Hospital, Capital Medical University, Neuroimaging Center, Beijing Neurosurgical Institute, No. 6 Tiantan Xili, Dongcheng District, Beijing, China, 100050; e-mail: djpbeijing@hotmail.com; or Tao Jiang, MD, Department of Neurosurgery, Beijing Tiantan Hospital, Capital Medical University, No. 6 Tiantan Xili, Dongcheng District, Beijing, China 100050; e-mail: jiangtao369@sohu.com.

Indicates open access to non-subscribers at www.ajnr.org

http://dx.doi.org/10.3174/ajnr.A2721
T1WI and T2WI and show heterogeneous enhancement on both contrast-enhanced CT scans and MR images. ${ }^{4}$ Histologically, hemangiopericytoma arises from pericytes of Zimmerman or pericapillary cells and can occur anywhere where capillaries are found. ${ }^{2,8,9}$ It has been classified as a mesenchymal tumor of the meninges and further subclassified into 2 histologic types, hemangiopericytoma and anaplastic hemangiopericytoma (corresponding to WHO grades II or III) by the WHO in 2007. ${ }^{10}$ The literature on CT and MR imaging of intracranial meningeal hemangiopericytomas is currently limited. To our knowledge, no large series of the imaging features of intracranial meningeal hemangiopericytomas in children and adolescents have been reported. The purpose of this report was to describe the CT and MR imaging findings in 9 cases of pathologically proved hemangiopericytomas as an aid to preoperative diagnosis.

\section{Case Series}

Between January 2003 and December 2010, 9 pathologically proved cases of intracranial meningeal hemangiopericytomas in children and adolescents were collected for review at the Beijing Tiantan Hospital. There were 5 males and 4 females, with ages ranging from 3 to 18 years (mean, 9.8 years; median, 10 years). All tumors underwent surgery followed by radiation therapy. The local institutional ethics committee approved this study and granted a waiver of informed consent. According to the WHO criteria, ${ }^{10}$ the tumor was subclassified as 1 of 2 histologic types, hemangiopericytoma or anaplastic hemangiopericytoma.

All precontrast CT examinations were performed on a 16-section CT scanner (Somatom Volume Zoom; Siemens, Erlangen, Germany) and on a 2-section CT scanner (HiSpeed; GE Healthcare, Milwaukee, Wisconsin). MR imaging sequences in 8 patients were acquired on a 3T MR imaging scanner (Genesis Signa; GE Healthcare) and in 1 patient by using a different 3T MR imaging scanner (Magnetom Trio; Siemens). Gd-DTPA $(0.2 \mathrm{~mL} / \mathrm{kg})$ was administered at a rate of 2.0 $\mathrm{mL} / \mathrm{s}$ through a 20 -gauge intravenous line with a power injector. All 


\begin{tabular}{|c|c|c|c|c|c|c|c|c|c|c|c|}
\hline Patient & Sex & $\begin{array}{l}\text { Age } \\
\text { (yr) }\end{array}$ & $\begin{array}{l}\text { Blood Loss } \\
\quad(\mathrm{mL})\end{array}$ & $\begin{array}{l}\text { Duration of } \\
\text { Symptoms } \\
\text { (mo) }\end{array}$ & Symptoms & $\begin{array}{l}\text { Extent of } \\
\text { Tumor } \\
\text { Removal }\end{array}$ & $\begin{array}{c}\text { Postoperative } \\
\text { Radiotherapy } \\
\text { (Gy) }\end{array}$ & $\begin{array}{c}\text { Follow-Up } \\
(\mathrm{mo})\end{array}$ & $\begin{array}{c}\text { Local } \\
\text { Recurrence }\end{array}$ & Metastases & Pathology \\
\hline$\overline{1}$ & $M$ & 3 & 400 & 0.5 & Seizures & CR & None & $95 / a$ & No & No & $\mathrm{AH}$ \\
\hline 2 & $\mathrm{~F}$ & 18 & 1000 & 0.5 & Headache & CR & 60 & $39 / a$ & No & No & $\mathrm{AH}$ \\
\hline 3 & $\mathrm{~F}$ & 10 & 4000 & 6 & Headache & CR & 60 & $36 / a$ & No & No & $\mathrm{H}$ \\
\hline 4 & $\mathrm{~F}$ & 17 & 1000 & 0.5 & Headache & CR & 60 & $29 / a$ & No & No & $\mathrm{AH}$ \\
\hline 5 & M & 3 & 350 & 0.3 & Headache & CR & None & $27 / a$ & No & No & $\mathrm{AH}$ \\
\hline 6 & M & 7 & 2000 & 12 & Seizures & CR & 60 & $16 / a$ & No & No & $\mathrm{AH}$ \\
\hline 7 & M & 17 & 13,000 & 3 & Headache & CR & 60 & $12 / a$ & No & No & $\mathrm{AH}$ \\
\hline 8 & M & 5 & 300 & 1.5 & Headache & CR & 60 & $8 / d$ & Yes & No & $\mathrm{AH}$ \\
\hline 9 & $\mathrm{~F}$ & 18 & 9000 & 12 & Headache & SR & 60 & $2 / a$ & No & No & $\mathrm{H}$ \\
\hline
\end{tabular}

a indicates alive; d, dead; CR, complete resection; SR, subtotal resection; $\mathrm{AH}$, anaplastic hemangiopericytoma; $H$, hemangiopericytoma

\begin{tabular}{lllllll}
\hline \multicolumn{2}{l}{ Table 2: General imaging features of $\mathbf{9}$ patients with hemangiopericytoma } \\
\hline \multicolumn{1}{c}{ Patient } & \multicolumn{1}{c}{ Location } & Shape & Size $^{\text {a }}(\mathrm{mm})$ & Necrosis & Edema & Hyperostosis or Bone Erosion \\
\hline 1 & Right middle cranial fossa & Multilobular & $55 \times 48 \times 41$ & Yes & Mild & Indeterminate \\
2 & Left frontal-temporal convexity & Multilobular & $60 \times 58 \times 55$ & Yes & Mild & Bone erosion of inner table \\
3 & Right middle cranial fossa & Multilobular & $67 \times 73 \times 72$ & Yes & None & Bone erosion \\
4 & Left frontal convexity & Multilobular & $50 \times 47 \times 51$ & Yes & Mild & Indeterminate, bone erosion \\
5 & Right occipital-parietal parasagittal & Multilobular & $54 \times 41 \times 46$ & Yes & Mild & No \\
6 & Right frontal parasagittal & Oval & $78 \times 76 \times 87$ & Yes & Moderate & No \\
7 & Bifrontal, anterior cranial fossa & Multilobular & $102 \times 72 \times 61$ & Yes & Mild & Indeterminate \\
8 & Right occipital-parietal convexity & Multilobular & $80 \times 80 \times 70$ & Yes & Moderate & Indeterminate, bone erosion \\
9 & Bioccipital-parietal parasagittal & Multilobular & $116 \times 66 \times 50$ & Yes & Moderate & No \\
\hline
\end{tabular}

a Length $\times$ width $\times$ height.

CT and MR examinations were performed only once in a patient within 2 weeks before surgery.

All 9 tumors were proved as hemangiopericytomas on initial pathologic review, and they were then histologically re-evaluated for the purposes of this retrospective study by a neuropathologist (Jun-mei Wang, 16 years of experience).

Four CT scans and $8 \mathrm{MR}$ examinations were reviewed. CT and MR imaging features evaluated on the neuroradiology studies were tumor location, shape, maximum diameter, CT attenuation, calcification, and hyperostosis or bone erosion on precontrast CT examination, signal intensity relative to cortical gray matter on T1WI and T2WI, degree of enhancement, homogeneity and pattern of enhancement, type of dural attachment, "dural tail" sign, necrosis or cystic degeneration, serpentine signal intensity voids of vessels, and presence and degree of edema as appropriate. Types of dural attachment were as follows: broad-based, with tumor diameter equal to or smaller than dural attachment; and narrow-based, with tumor diameter greater than dural attachment. Degree of edema was as follows: mild edema, edema volume much smaller than the volume of the tumor; and moderate edema, edema volume roughly equal to or greater than the volume of the tumor.

Manual measurement of the maximum tumor diameter (in millimeters) was performed in the anteroposterior, left-right, and cephalocaudal directions by using the scanner console software. For each tumor, the measurement was performed twice, and the average value was recorded as the final diameter.

All CT and MR images were independently evaluated by 2 neuroradiologists (Qian Chen, 16 years of experience and Xu-zhu Chen, 15 years of experience). They were blinded to each other's evaluations and to clinical and pathologic information. Consensus was established when discrepancies were present. The clinical characteristics and imaging findings are summarized in Tables 1 and 2.

The most common presenting symptom in our series was head- ache in $78 \%(7 / 9)$ of patients, followed by seizures in $22 \%(2 / 9)$ of patients. The intraoperative blood loss ranged from 300 to $13,000 \mathrm{~mL}$ (mean, $3561 \mathrm{~mL}$; median, $1000 \mathrm{~mL}$ ). The mean duration of symptoms was 4.0 months, and the median was 1.5 months, with a range of 0.3 to 12 months.

All tumors in this study were supratentorial. The most common locations were the convexity (3/9) and the parasagittal region (3/9). The maximum diameter ranged from 51 to $116 \mathrm{~mm}$, and the mean was $75 \mathrm{~mm}$. Eight tumors were irregularly multilobular, and the remaining tumor was oval. Calcification with bone erosion was only seen in 1 tumor (Fig 1). Erosion of the adjacent bone was noted in $22 \%(2 / 9)$ of patients, indeterminate bone erosion was noted in $22 \%$ (2/9) of patients, whereas no hyperostosis was noted in any patients. Peritumoral edema was seen in 8 patients on CT images, MR images, or both, with either a mild degree ( 5 cases) or moderate degree (3 cases). Edema was not seen in 1 patient.

On precontrast CT examinations, 2 of 4 cases showed mixed low, iso-, and high attenuation, and an additional 2 cases showed mixed low and isoattenuation related to cortical gray matter.

On MR examinations, 5 of 8 cases showed mixed hypo-, iso-, and hyperintensity, 3 cases showed mixed hypo- and isointensity relative to cortical gray matter on T1-weighted precontrast images; 7 cases showed mixed hypo-, iso-, and hyperintensity, 1 case showed mixed iso- and hyperintensity relative to cortical gray matter on T2WI. All 7 cases showed marked and heterogeneous enhancement on T1WI (Figs 2 and 3). An obvious dural attachment of the tumor was noted in all 7 postcontrast MR examinations. One of 7 cases showed broadbased dural attachment that was characterized as a hemangiopericytoma; 6 of 7 cases showed narrow-based dural attachment, and all were characterized as anaplastic hemangiopericytomas. Four of 7 cases that underwent postcontrast MR examinations showed the "dural tail" sign, 1 was a hemangiopericytoma and 3 were anaplastic hemangiopericytomas. Three of 7 cases lacked the "dural tail" sign and 

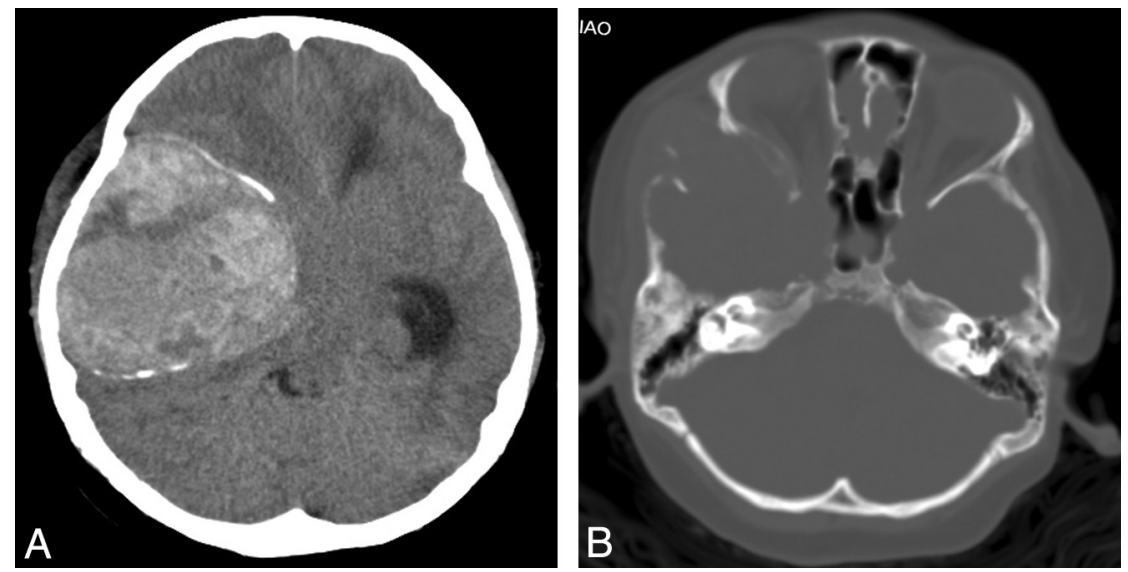

Fig 1. Patient 3. Hemangiopericytoma with peripheral calcification and bone erosion. A, Axial CT examination shows a mass over the right middle cranial fossa with upward extension to the temporal lobe. The mass shows mixed low, iso-, and high attenuation with peripheral calcification. $B$, Axial CT (bone window) shows destruction of right temporal bone and lateral wall of right orbit.
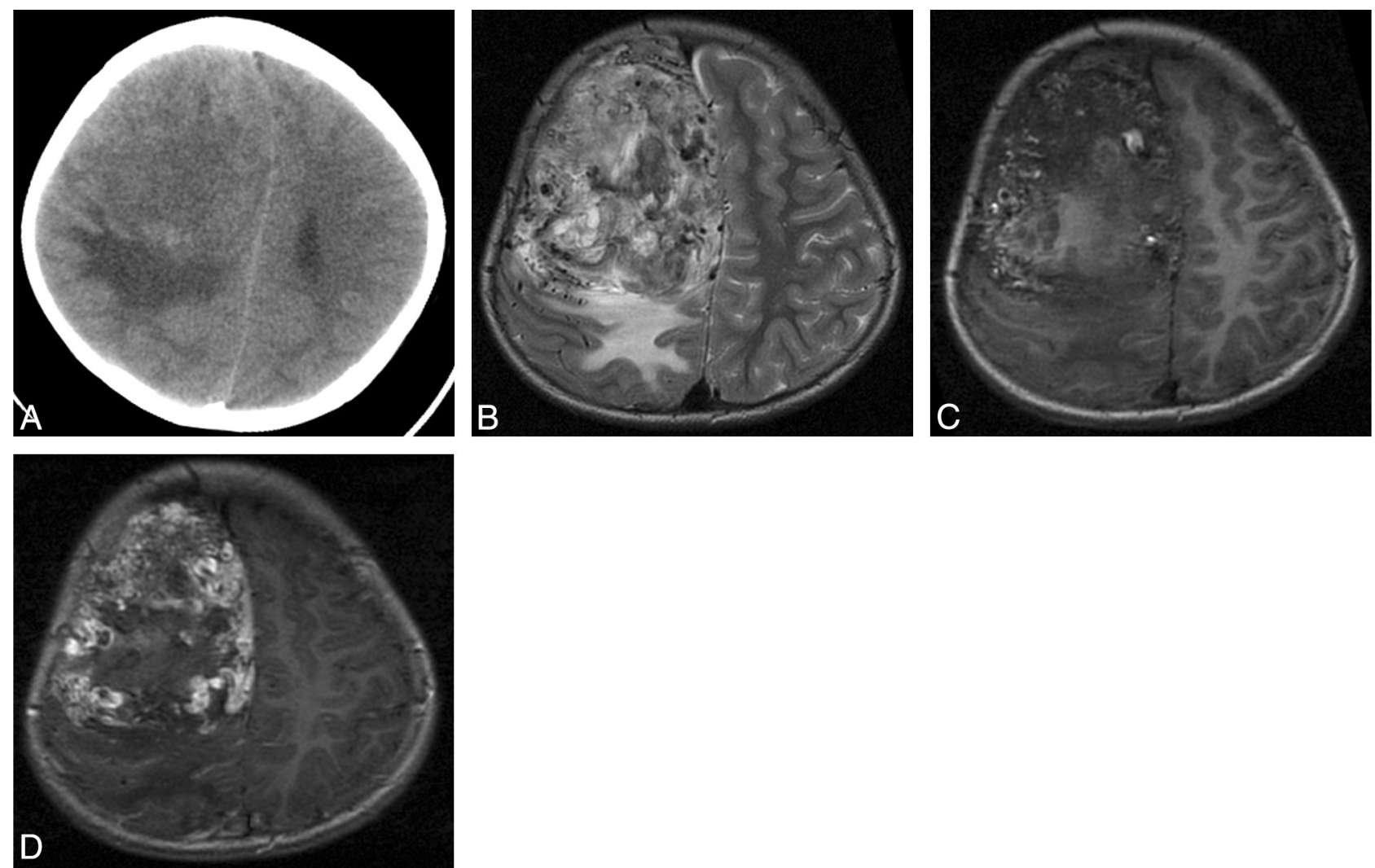

Fig 2. Patient 6. Anaplastic hemangiopericytoma with narrow-based dural attachment and AVM-like features. $A$, Axial CT examination shows a mass located in the right frontal parasagittal convexity. The mass shows mixed low and isoattenuation with moderate surrounding edema. $B$ and $C$, Mass shows mixed hypo-, iso-, and hyperintensity with AVM-like signal intensity flow voids on precontrast axial T2 and T1-weighted images. D, Mass shows marked and heterogeneous enhancement with narrow-based dural attachment on postcontrast axial T1-weighted images but lacks the "dural tail" sign.

were characterized as anaplastic hemangiopericytomas. There was necrotic or cystic signal intensity in all cases of our series, later proved by pathology. Eight tumors had serpentine signal intensity voids in vessels. AVM-like signal intensity flow voids were observed on MR images of 2 tumors.

\section{Discussion}

In our series, precontrast CT images showed heterogeneous attenuation, compared with a study of meningioma in children where the solid component of the tumor showed hy- perattenuation relative to the cortical gray matter before enhancement. ${ }^{11}$ In our study, hemangiopericytoma with peripheral calcification was seen in a 10 -year-old girl (patient 3 ) on CT examination. This finding contrasted with other studies in which calcification was not seen in hemangiopericytoma. ${ }^{2,4}$ Calcification in meningeal hemangiopericytoma has rarely been reported; indeed, we could find only 4 cases in 3 studies $^{12-14}$ : a 67-year-old woman, a 23-year-old woman, a 6 -week-old boy, and a 12-year-old girl. Calcifications in these 5 cases (including the patient in our study) were all located in 

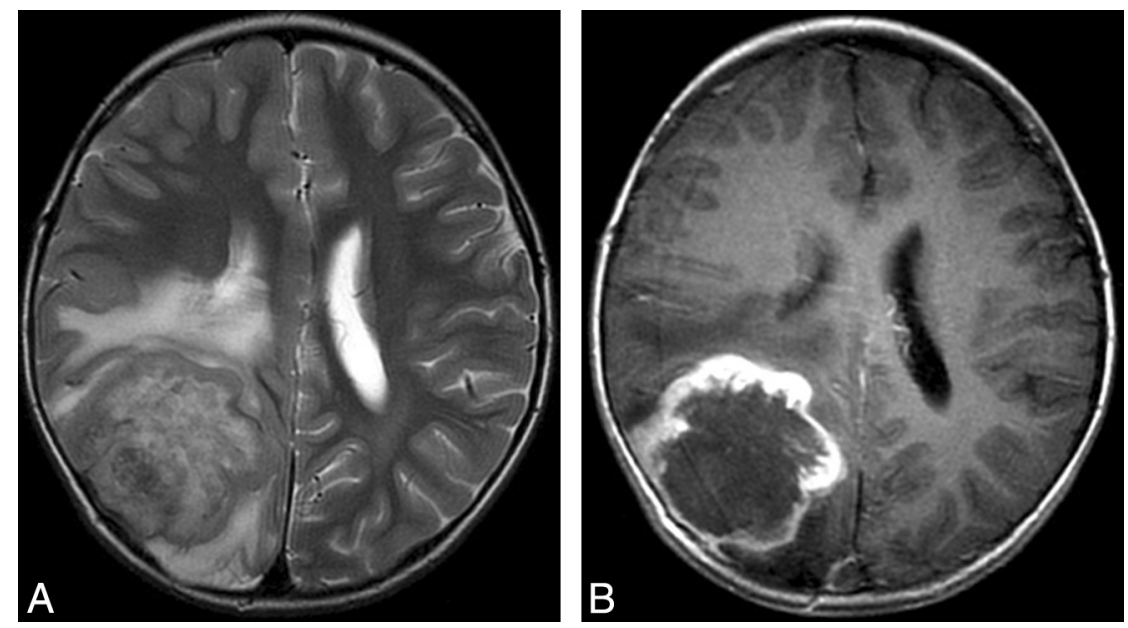

Fig 3. Patient 9 . Hemangiopericytoma with the "dural tail" sign. $A$, Mass shows mixed hypo-, iso-, and hyperintensity on precontrast axial T2-weighted images. $B$, Mass shows marked and heterogeneous enhancement with narrow-based dural attachment on postcontrast axial T1-weighted images and shows the "dural tail" sign.

the tumor periphery. Furthermore, it is notable that 4 of 5 cases were females. Besides calcification, there also was bone erosion in our patient, as had occurred in the previously studied 12-year-old girl. ${ }^{13}$ Bone erosion in meningeal hemangiopericytoma has been reported in the literature. ${ }^{2,4}$ Tumor calcification, bony hyperostosis, and calvarial thinning are frequent in childhood meningiomas. ${ }^{15,16} \mathrm{Im}$ et al ${ }^{16}$ reported tumor calcifications in childhood meningiomas that ranged in appearance from fine and cloudlike to attenuated and homogeneously nodular.

The intraoperative blood loss was large in those patients with intracranial meningeal hemangiopericytomas. It has been recommended that preoperative embolization be performed. ${ }^{7}$ Large quantities of blood should be prepared for transfusion during surgery. Tumors in our study were irregularly multilobular extra-axial hypervascular tumors. In all 8 studied tumors, multiple signal intensity voids of vessels were observed on MR imaging. In addition, in 2 tumors, AVM-like signal intensity flow void was observed on MR imaging, which has not been reported in the literature. Therefore, when an irregularly multilobular extra-axial tumor shows multiple signal intensity voids of vessels, and AVM-like signal intensity flow voids in particular are seen on MR imaging, the diagnosis of hemangiopericytoma should be considered.

Chiechi et $\mathrm{al}^{4}$ reported that one-third of the hemangiopericytomas that they studied showed a narrow-based dural attachment, and they found no relationship between histologic subtype of hemangiopericytoma and the mode of dural attachment. In our series, 6 of 7 tumors with narrow-based dural attachment imaged by contrast MR were all anaplastic hemangiopericytomas. Buetow et $\mathrm{al}^{17}$ reported that up to $85 \%$ of meningiomas studied in their series showed a broad base of dural attachment. Im et $\mathrm{al}^{16}$ reported that a dural attachment was not a common sign in childhood meningioma. In our series, we found that the average duration of symptoms with hemangiopericytoma was 4.0 months, whereas in pediatric patients, the average duration with meningioma was 1.2 years. $^{18}$

Besides intracranial meningioma, the following tumors should be differentiated. 1) Glioblastoma. This can occur anywhere in the brain but is generally characterized by in- volvement of white matter and can spread across the corpus callosum to the contralateral hemisphere, whereas glioblastoma is rare in children. The tumor boundary in glioblastoma is not clear because of this tumor's widely infiltrative nature. ${ }^{19}$ 2) AT/RTs. Meyers et $\mathrm{al}^{20}$ reported that at the time of diagnosis, patients with AT/RTs had a mean age of 3.7 years. In our series, the maximum diameter at presentation with hemangiopericytoma was $>51 \mathrm{~mm}$, whereas only 3 of 13 AT/RTs cases in the Meyers et al study had a maximum diameter $>40 \mathrm{~mm}$. It would be difficult to differentiate AT/RT from intracranial meningeal hemangiopericytoma on CT and MR imaging if the AT/RT had an extra-axial location, because AT/RT also shows mixed signal intensity on MR and mixed attenuation on CT. ${ }^{20}$ 3) PNET. Khong et $\mathrm{al}^{21}$ reported a 3-year-old girl with a supratentorial PNET of the dura mater that showed mixed isoand low attenuation with bone erosion on precontrast CT images and heterogeneous contrast enhancement with linear enhancing septa on contrast CT images. Except for the lack of calcification, this case was similar to patient 3 in our series. 4) Ependymoma. Extra-axial ependymoma is another extremely rare intracranial tumor. Only a few cases have been recorded in the literature. ${ }^{22,23}$ It is very difficult to differentiate extra-axial ependymoma from hemangiopericytoma by radiologic findings alone.

There are some limitations to our report. First, because of the small incidence of intracranial meningeal hemangiopericytoma, we could only review a small number of cases. Second, the data were retrospectively evaluated. Finally, there were no postcontrast CT images available, and more importantly, no digital subtraction angiography images. Subsequently, no assessment of the feeding arteries was done.

\section{Conclusions}

Children and adolescents with intracranial meningeal hemangiopericytomas have a short duration ( $<12$ months) of symptoms. Intracranial meningeal hemangiopericytomas in children and adolescents are also prone to bleeding during surgery. The results of this report suggest that CT and MR imaging findings may be helpful for preoperative diagnosis. Hemangiopericytoma should be considered where radiologic findings show an extra-axial tumor of large size $(>51 \mathrm{~mm})$; 
mixed necrotic and cystic attenuation and/or signal intensity; marked heterogeneous enhancement; multiple signal intensity flow voids of vessels, especially AVM-like signal intensity flow voids seen on MR imaging; and possible association with narrow-based dural attachment and bone erosion and peripheral calcification but lacking hyperostosis.

\section{References}

1. Stout AP, Murray MR. Hemangiopericytoma: a vascular tumor featuring Zimmerman's pericytes. Ann Surg 1942;116:26-33

2. Guthrie BL, Ebersold MJ, Scheithauer BW, et al. Meningeal hemangiopericytomas: histopathological features, treatment and long-term follow-up of 44 cases. Neurosurgery 1989;25:514-22

3. Herzog CE, Leeds NE, Bruner JM, et al. Intracranial hemangiopericytoma in children. Pediatr Neurosurg 1995;22:274-79

4. Chiechi MV, Smirniotopoulos JG, Mena H. Intracranial hemangiopericytomas: MR and CT features. AJNR Am J Neuroradiol 1996;17:1365-71

5. Peace RJ. A congenital neoplasm of the brain of a newborn infant: report of a case with necropsy. Am J Clin Pathol 1954;24:1272-75

6. Mena H, Ribas JL, Pezesk GH, et al. Hemangiopericytoma of the central nervous system: a review of 94 cases. Hum Pathol 1991;22:84-91

7. Fountas KN, Kapsalaki E, Kassam M, et al. Management of intracranial meningeal hemangiopericytomas: outcome and experience. Neurosurg Rev 2006; 29:145-53

8. Rutkowski MJ, Sughrue ME, Kane AJ, et al. Predictors of mortality following treatment of intracranial hemangiopericytoma. J Neurosurg 2010;113:333-39

9. Fitzpatrick D, Mahajan J, Lewkowitz M, et al. Intradural hemangiopericytoma of the lumbar spine: a rare entity. AJNR Am J Neuroradiol 2009;30:152-54

10. Louis DN, Ohgaki H, Wiestler OD, et al. The 2007 WHO Classification of Tumours of the Central Nervous System. Acta Neuropathol 2007;114:97-109
11. Darling CF, Byrd SE, Mugica MR, et al. MR of paediatric intracranial meningiomas. AJNR Am J Neuroradiol 1994;15:435-44

12. Ruscalleda J, Feliciani M, Avila A, et al. Neuroradiological features of intracranial and intraorbital meningeal haemangiopericytomas. Neuroradiology 1994;36:440-45

13. Cole JC, Naul LG. Intracranial infantile hemangiopericytoma. Pediatr Radiol 2000;30:271-73

14. Alen JF, Lobato RD, Gomez PA, et al. Intracranial hemangiopericytoma: study of 12 cases. Acta Neurochir (Wien) 2001;143:575-86

15. Amirjamshidi A, Mehrazin M,Abbassioun K. Meningiomas of the central nervous system occurring below the age of 17: report of 24 cases not associated with neurofibromatosis and review of literature. Childs Nerv Syst 2000;16: 406-16

16. Im SH, Wang KC, Kim SK, et al. Childhood meningioma: unusual location, atypical radiological findings, and favorable treatment outcome. Childs Nerv Syst 2001;17:656-62

17. Buetow MP, Buetow PC, Smirniotopoulos JG. From the archives of the AFIP: typical, atypical, and misleading features in meningioma. Radiographics 1991 11:1087-106

18. Caroli E, Russillo M, Ferrante L. Intracranial meningiomas in children: of 27 new cases and critical analysis of $\mathbf{4 4 0}$ cases reported in the literature. J Child Neurol 2006;21:31-36

19. Rickert $\mathrm{CH}$, Paulus W. Epidemiology of central nervous system tumors in childhood and adolescence-based on the new WHO classification. Childs Nerv Syst 2001;17:503-11

20. Meyers SP, Khademian ZP, Biegel JA, et al. Primary intracranial atypical teratoid/rhabdoid tumors of infancy and childhood: MRI features and patien outcomes. AJNR Am J Neuroradiol 2006;27:962-71

21. Khong PL, Chan GC, Shek TW, et al. Imaging of peripheral PNET: common and uncommon locations. Clin Radiol 2002;57:272-77

22. Andrew S. Youkilis PP, Paul E. Parasagittal ependymoma resembling falcine meningioma. AJNR Am J Neuroradiol 2001;22:1105-08

23. Goto T, Ohata K, Tsuyuguchi N, et al. Extra-axial subarachnoid ependymoma of the cerebral convexity. Acta Neurochir (Wien) 2003;145:913-17 